\title{
Text Conventions
}

/ Point at which overlapping speech begins

// Point at which two or more characters begin to speak the same words simultaneously

Italics Stage directions

\section{Note}

When AmY, BEN and WeNDY speak alone, they directly address the audience. When they speak synchronously with another character, they physically shadow the character and address the character's addressee. They also adopt the intonation of the character whose speech they share. 